\title{
Reduction of the EM Backscattering for Frequency-Independent Stealth Coating
}

\author{
Salah I. Yahya ${ }^{\# 1}$, Yazen A. Khalil ${ }^{\# 2}$ \\ \# Department of Software Engineering, Faculty of Engineering, Koya University \\ Danielle Mitterrand Boulevard, Koya KOY45, Kurdistan Region - Iraq
}

\begin{abstract}
This paper presents a frequency-independent reduction of the backscattering technique for stealth coating applications. Although the proper shape of the target may play a role in reduction of the backscattering as a goal, choosing absorbing materials having proper electrical and magnetic properties with a certain matching technique may achieve this goal. A ferrite-titanate material with thickness of $3 \mathrm{~mm}$ is suggested for coating a target with a technique explained in the paper to attain reduction in backscattering above $1 \mathrm{GHz}$. The proposed technique together with the material may find a good chance in stealth technology, for both military and non-military applications.
\end{abstract}

Keywords - Backscattering, stealth technology, space cloth, matching, complex permittivity, complex permeability.

\section{INTRODUCTION}

Reduction of the backscattering from an object in stealth technology is to reduce the likelihood of betraying its presence and to minimize the probability of its detection when active search and tracking techniques are employed [1]. Webster defines stealth as "the act of going furtively or as a secret procedure or action." Stealthy is defined as "accomplished secretly or furtively or as acting clandestinely, furtive, or sly." Stealth technology also termed LO technology (low observable technology) is a sub-discipline of military tactics and passive electronic countermeasures [2]. Stealth technology is generally associated with aircraft, however this technology also receives increasingly more attention in ships, submarine, tanks and satellite designs. Nowadays more attention is paying for stealth technology that associated with non-military applications, e.g. wind turbine [3].

A considerable reduction of the object, e.g., aircraft stealth, back-scattering can be accomplished by two ways;

1. Reflect the incident radar electromagnetic wave (EMW) into any direction other than back to the radar site

2. Utilize radar absorbing material to turn the radar illumination energy into heat.

The second way is the main goal of this paper where a frequency-independent coating material technique is suggested for stealth technology.

\section{General CONSIDERATION}

Any mass object embedded into free-space represents a non-homogeneity causing the scattering of electromagnetic waves. The wave reflected in the direction of the incident wave is called the back-scattered wave. This effect is in detail studied in radar engineering where the property of the scattering target is described by means of the target crosssection [4];

$$
\sigma=\frac{P_{S C}}{S_{I N C}}
$$

where $\mathrm{P}_{\mathrm{SC}}$ is the total power scattered isotropically and $\mathrm{S}_{\mathrm{INC}}$ is the power density of the incident wave at the location of the target. The highest reflection is caused by conducting targets. For example the perfectly conducting sphere has the crosssection equal to [4]:

$$
\sigma=\pi a^{2}
$$

where a is the radius of the sphere. If the sphere is made of imperfectly conducting material then the radar cross-section becomes smaller, e.g. at frequency of $300 \mathrm{MHz}$ the radar cross-section of the moon is 10 times smaller than the crosssection of the equivalent perfectly conducting.

Except the sphere which is an isotropic scatterer, the wave scattered back by all geometrical bodies depends on their orientation with respect to the incident wave. It is quite obvious that the perfectly conducting plane which behaves like a mirror for electromagnetic waves will reflect back the maximum power only if it is perpendicular to the incident wave. On the other hand there is no back-scattered wave if the conducting plane is inclined to the incident wave. From this point of view the perfectly conducting cube with the body diagonal perpendicular to the incident wave has the target cross-section near to zero.

\section{SPECIFICATION OF THE GOAL}

It follows the previous section that there are several factors which may reduce the back-scattered electromagnetic wave:

\section{A. The Proper Shape of the Target}

It means that its surface should be composed of plane areas inclined to the direction of incident wave. This is a job for designers of a particular device representing the target and will not be discussed in this paper in details. But even if the target which is usually made of high conducting material has the proper shape being in one particular positions with respect to the incident wave, we can never exclude the case that by changing the orientation some of the target plant wall happens to be directed perpendicularly to incident wave. Fig. 1 shows the two scenarios of using Cubic and spherical shape targets. It is obvious that always there is a reflection from the spherical shape target regardless the position, whereas, at 
certain position the cubic shape target may not reflect the EMW in the direction perpendicularly to incident wave. This principle in shaping the stealth targets is already applied practically as shown in Fig. 2, where the reflection direction affected by the target shape, e.g., passenger airplane and stealth fighter.

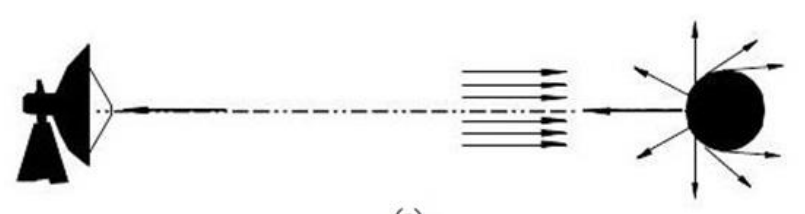

(a)

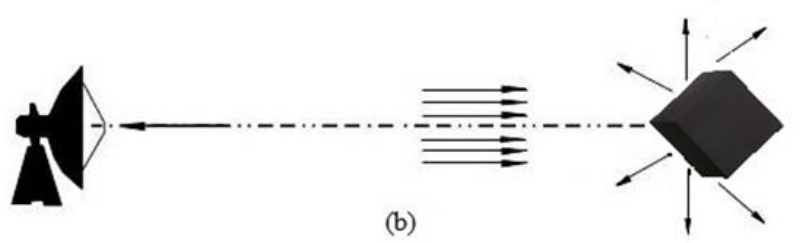

Fig. 1. The reflection directions of radar incident waves on (a) spherical shape target and (b) cubic shape target.

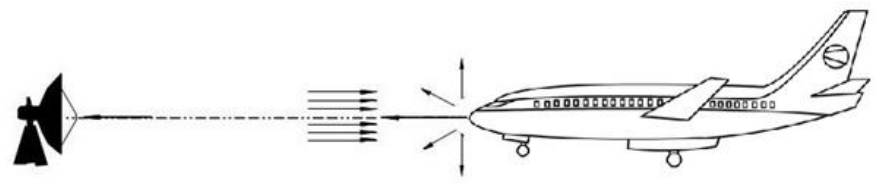

(a)

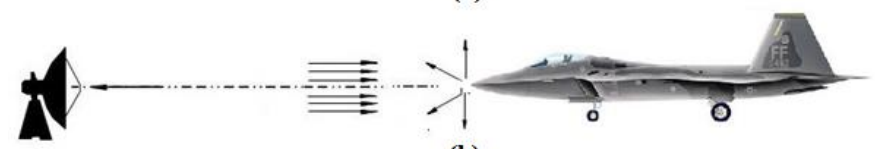

(b)

Fig. 2. An application of Fig. 1 shows the reflection directions of radar incident waves on (a) passenger airplane and (b) stealth fighter.

\section{B. Choosing the Proper Material}

The target itself should be made (at least into the depth of penetration of electromagnetic wave) of lossy material which absorbs a part or even the whole energy of the electromagnetic wave. But even if we use the proper material which absorbs the incident wave energy the reflection from the surface will appear. It means that the third condition must be satisfied.

\section{Matching between the Absorbing Structure and Free space}

It means that the main goal of the paper is to find how to reduce the reflection from the conducting plane when the incident electromagnetic wave impacts the plane perpendicularly.

\section{REFLECTION FROM MULTI-LAYER SYSTEM}

\section{A. The General Theory}

The general model of the structure which may reduce the reflection of the plane wave normally incident is the multilayer system consisting on $n$ regions each described by material $\varepsilon, \mu$ and $\sigma$ as shown in Fig. 3, [5]

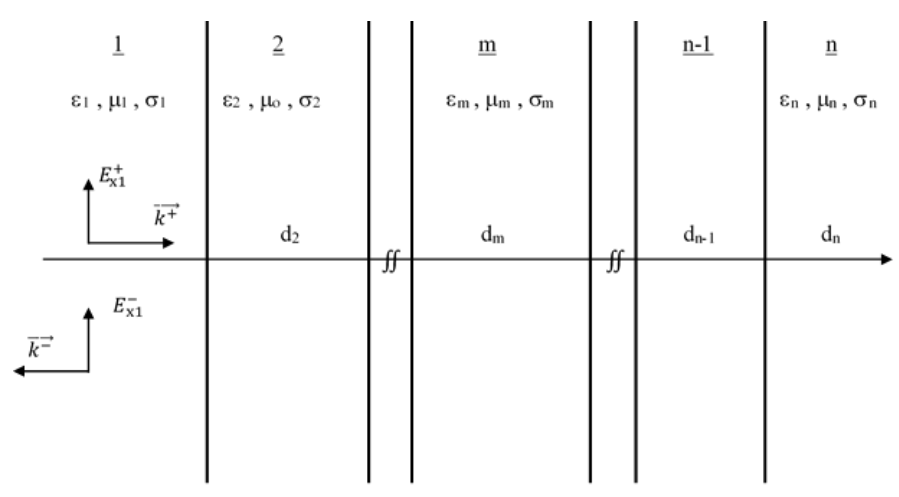

Fig. 3. The multi-layer system.

In each region, say $m$, except the last one, the total electric field consists of two components: the forward-traveling one with amplitudes $E_{m}^{+}$and $E_{m}^{-}$respectively:

$$
\begin{gathered}
E_{x m}(z)=E_{m}^{+} e^{-\gamma_{m} z}+E_{m}^{-} e^{+\gamma_{m} z}=E_{m}^{+} e^{-\gamma_{m} z}[1+ \\
\left.\Gamma_{\mathrm{m}}(\mathrm{z})\right] \\
\Gamma_{m}(z)=\frac{E_{m}^{-}}{E_{m}^{+}} e^{2 \gamma_{m} z} \\
\gamma_{m}=\alpha_{m}+j \beta_{m}
\end{gathered}
$$

Where $\Gamma$ is the reflection coefficient and $\gamma$ is the complex propagation constant. The subscript $m$ shows that all these quantities belong to the region $m$. the real part of Equation (5) is the attenuation constant.

$$
\alpha_{m}=\omega \frac{\sqrt{s_{m} \mu_{m}}}{\sqrt{2}}\left[\sqrt{1+\left(\frac{\sigma_{m}}{\omega s_{m}}\right)^{2}}-1\right]
$$

and the imaginary part (4) is the phase constant

$$
\beta_{m}=\omega \cdot \frac{\sqrt{s_{m} \mu_{m}}}{\sqrt{2}}\left[\sqrt{1+\left(\frac{\sigma_{m}}{\omega s_{m}}\right)^{2}}+1\right]
$$

Without loss of the generality we have assumed the electric field in the direction of $x$ axis so that the corresponding magnetic field intensity is:

$$
\begin{aligned}
& H_{y m}(z)=H_{m}^{+} e^{-\gamma_{m} z}+H_{m}^{-} e^{+\gamma_{m} z}=\frac{E_{m}^{+}}{\eta_{m}} e^{-\gamma_{m} z}[1- \\
& \left.\Gamma_{m}(\mathrm{z})\right] \\
& \quad \eta_{m}=\frac{E_{m}^{+}}{H_{m}^{+}}=-\frac{E_{m}^{-}}{H_{m}^{-}}=\sqrt{\frac{\mu_{m}}{s_{m}-j \frac{\sigma_{m}}{\omega}}}
\end{aligned}
$$


where $\eta$ is the intrinsic impedance of the $m$-th region.

A total field impedance at any $\mathrm{z}$-coordinate in the $\mathrm{m}$-th region is given by the expression.

$$
Z_{m}=\frac{E_{x m}(z)}{H_{y m}(z)}=\eta_{m} \frac{1+\Gamma_{m}(z)}{1-\Gamma_{m}(z)}
$$

A converse expression to Equation (10) is obtained by solving it for $\Gamma_{m}(z)$

$$
\Gamma_{m}(z)=\frac{z_{m}(z)-\eta_{m}}{z_{m}(z)+\eta_{m}}
$$

If we know $\Gamma_{m}$ at the particular coordinate $z$ then any other location with coordinate $z$ its value is

$$
\Gamma(z)=\Gamma(\hat{z}) e^{2 \gamma(z-z)}
$$

Using these expressions we can recalculate the impedance from one point $\bar{z}$ equal to $Z_{m}(\dot{z})$ to the other point with coordinate $z$ :

$$
Z_{m}(z)=\eta_{m} \frac{z_{m}(z)+\eta_{m} \tanh \gamma_{m}(z-z)}{\eta_{m}+z_{m} \tanh \gamma_{m}(z-z)}
$$

Whereas the reflection coefficient is discontinuous at the any interface between different regions, the impedance $Z_{m}$ be continuous across the interface which follows from the boundary conditions for electric and magnetic field intensity.

A special case appears when the impedance at $\bar{z}$ is equal to zero which corresponds to the perfectly conducting plane placed at $\mathbf{z}$

$$
Z_{m}(z)=\eta_{m} \tanh \gamma_{m}(z-z)
$$

Another special case can be obtained by simulating the infinite impedance at the location $\bar{z}$ which gives the impedance

$$
Z_{m}(z)=\eta_{m} \frac{1}{\tanh \gamma_{m}(z-z)}=\eta_{m} \operatorname{coth} \gamma_{m}(z-z)
$$

All the quantities in previous are generally complex numbers or complex functions. As in the case of the multilayer structure perpendicular to the direction of the propagation of the electromagnetic wave no transverse direction is preferred, we can assume without the loss of generality that electric field intensity is in the direction of the $\mathrm{X}$-axis.

\section{B. Region with Conducting Material}

Any medium is considered as a good conductor if, [5]

$$
\frac{\sigma_{m}}{\omega s_{m}} \gg 1
$$

Equations (5) and (6) then can be reduced to

$$
\alpha_{m}=\beta_{m}=\sqrt{\frac{\omega \mu_{m} \sigma_{m}}{2}}
$$

and the intrinsic impedance of the medium becomes

$$
\eta_{m}=(1+j) \sqrt{\frac{\omega \mu_{m}}{2 \sigma_{m}}}
$$

The inverse value of $\alpha_{m}$ defines the depth of penetration

$$
\delta_{m}=\frac{1}{\alpha_{m}} \sqrt{\frac{2}{\omega \mu_{m} \sigma_{m}}}
$$

For an ideal case of a perfect conductor we get the following values:

$$
\begin{array}{crl}
\alpha_{m}=\beta_{m} \rightarrow \infty, & \eta_{m} & \rightarrow 0, \\
\delta_{m} \rightarrow 0, & \Gamma_{m}(0) & =-1
\end{array}
$$

\section{PARALlEL IMPEDANCE MATCHING}

\section{Transmission Line Analogy}

Comparing all derived formulae with formulae describing propagation along the transmission lines we can see that they are very similar. This suggests that some techniques used in transmission lines may be used also for multilayer systems.

Let us consider the matching by means of the stub tuner shown in Fig. 4-a. The load impedance $Z_{L}$ is transformed by the transmission line of length $d$ into the impedance $Z_{\text {in }}$. The length of the line $d$ together with the parallel impedance of the stub $Z_{s t}$ are chosen to give the characteristics impedance of the transmission line $Z_{0}$.

Then $Z_{\circ}$ is given by the following relation;

$$
Z_{0}=\frac{1}{\frac{1}{Z_{\text {in }}}+\frac{1}{Z_{s t}}}
$$

In this case the load is matched to the transmission line and no reflection appears.

The principle of parallel impedance matching can be used also in the case when the load impedance is equal to zero; it means in case when the transmission line is short-circuited as shown in Fig. 4-b. The transmission line of length $l=\lambda / 4$ transforms the zero load impedance into the infinite impedance which is in parallel to the impedance $Z_{o}=Z_{p}$ so that the resulting impedance is equal to the characteristic impedance of the line and no reflection appears. 


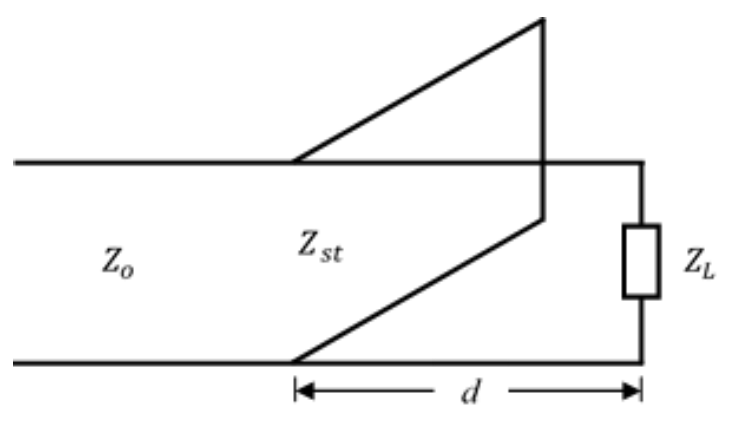

(a)

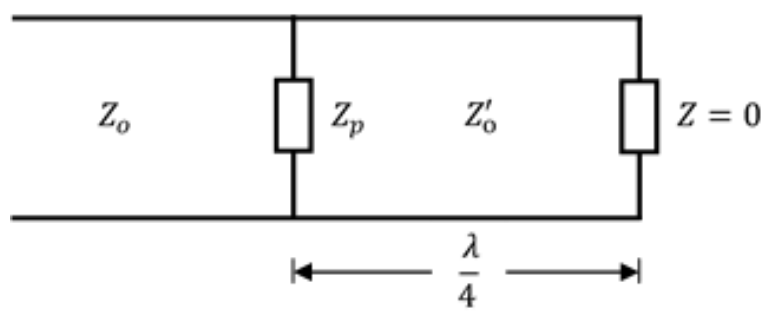

(b)

Fig. 4. The parallel impedance matching configuration.

\section{The Space Cloth}

Now we shall apply the same method to the four-region system of parallel layers as shown in Fig. 5. Despite all regions are in series it can be shown that, surprisingly, under some condition one layer behaves like connected in parallel to the system. Region 4 is a perfect conductor which represents the zero impedance medium for electromagnetic wave coming from the left. Region 3 is filled by lossless dielectric layer of the thickness $d=1 / 4$ which transforms, according to Equation (14), the short-circuit to the infinite impedance at the interface between region 2 and 3. Region 2 is made of a good but not perfect conducting material with thickness $h$ and intrinsic impedance given by (18):

$$
\eta_{2}=(1+j) \sqrt{\frac{\mu_{0} \omega}{2 \sigma_{2}}}
$$

The infinite impedance from the interface 2-3 is transformed to the interface 1-2 by means of Equation (15):

$$
Z_{2}=\eta_{2} \frac{1}{\tanh \gamma_{2} h}
$$

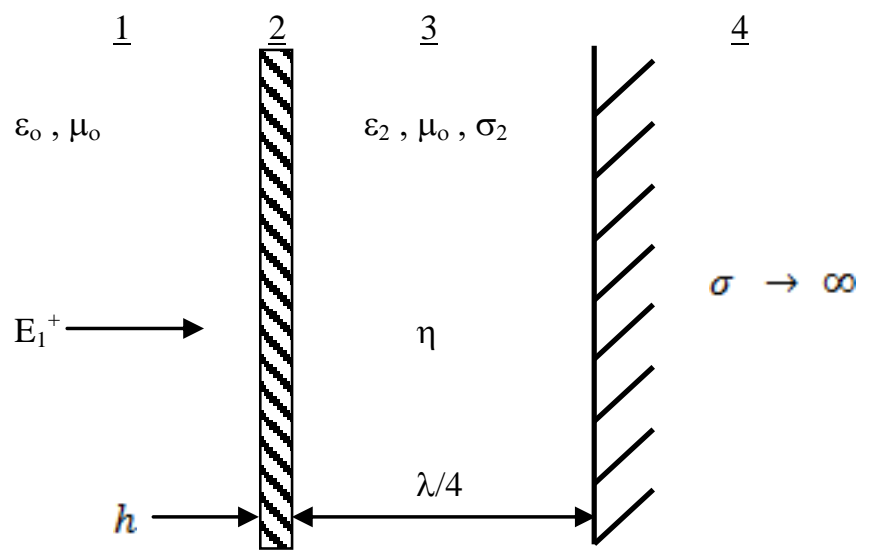

Fig. 5. The space cloth configuration.

Assuming that the thickness $h$ is very small we can use the approximation $\tanh x \cong x$ so that

$$
Z_{2}=\eta_{2} \frac{1}{\gamma_{2} h}=(1+j) \sqrt{\frac{\omega \mu_{0}}{2 \sigma_{2}}} \frac{1}{(1+j) h \sqrt{\frac{\omega \mu_{0 \sigma_{2}}}{2}}}=\frac{1}{\sigma_{2} h}
$$

Now we can choose the values of $\square_{2}$ and $h$ in such a way that

$$
\sigma_{2} h=\frac{1}{120 \pi}=\frac{1}{\eta_{0}}
$$

Then the input impedance of region 2 is equal to intrinsic impedance of free space and no reflection appears. The very thin layer of conducting material behaves like parallel impedance and absorbs the energy of incoming wave. Because of its very small thickness it is usually called the space paper or the space cloth.

As a practical example and based on Fig. 5, by choosing a lossless dielectric material, region 3 , with $\varepsilon_{r}=4$ at frequency of $9 \mathrm{GHz}$;

$\eta_{\mathrm{o}}=120 \pi, \lambda=\frac{3 \times 10^{8}}{f}$
$d_{3}=\frac{\lambda}{4} 10^{3}=8.333 \mathrm{~mm}$

The following materials can be used the space cloth with thickness calculated for each material as shown below:

1) with Carbon material

$$
\begin{aligned}
& \sigma_{1}=3 \times 10^{4}, h=\frac{1}{\sigma_{1} \eta_{0}} \times 10^{3}, \\
& h=8.838 \times 10^{-5} \mathrm{~mm}
\end{aligned}
$$

2) With Graphic material

$$
\begin{aligned}
& \sigma_{2}=10^{5}, \quad h=\frac{1}{\sigma_{2} \eta_{0}} \times 10^{3}, \\
& h=2.652 \times 10^{-5} \mathrm{~mm}
\end{aligned}
$$




\section{FREQUENCY-INDEPENDENT METHOD}

The method described in section $\mathrm{V}$ has one substantial drawback - it works perfectly only for one particular frequency. To avoid this frequency dependence, we must not use any effect which depends on frequency like for example the superposition of wave reflected from the front and from the back interface of any dielectric layer which depends on the thickness expressed in wavelength.

\section{A. The Brief Theoretical Online}

In Section IV we found that the impedance which appears in a lossy medium backed by perfectly conducting layer is given by Equation (14):

$$
Z(z)=\eta \cdot \tanh (\tau z)
$$

Where $\eta$ is the intrinsic impedance of the medium, Equation (9), and $\tau$ is the complex propagation constant.

For particular values listed below, Fig. 6 shows the impedance of the lossy medium versus its thickness.

$$
\begin{aligned}
& \varepsilon_{o}=\frac{10^{-9}}{36 \pi} \quad \text { and } \mu_{o}=4 \pi \times 10^{-7} \\
& \mu_{r}=1 \text { and } \varepsilon_{r}=3 \\
& f=10^{10}, \sigma=0.5, \omega=2 \pi f \text { and } Z_{o}=120 \pi \\
& Z_{d}=\left|Z_{o} \sqrt{\frac{\mu_{r}}{\varepsilon_{r}-i\left(\frac{\sigma}{\omega-\varepsilon_{o}}\right)}}\right|=213.017
\end{aligned}
$$

Using PTC ${ }^{(8)}$ MathCAD, the impedance given in Equation (14) can be sketched as a function of the medium thickness

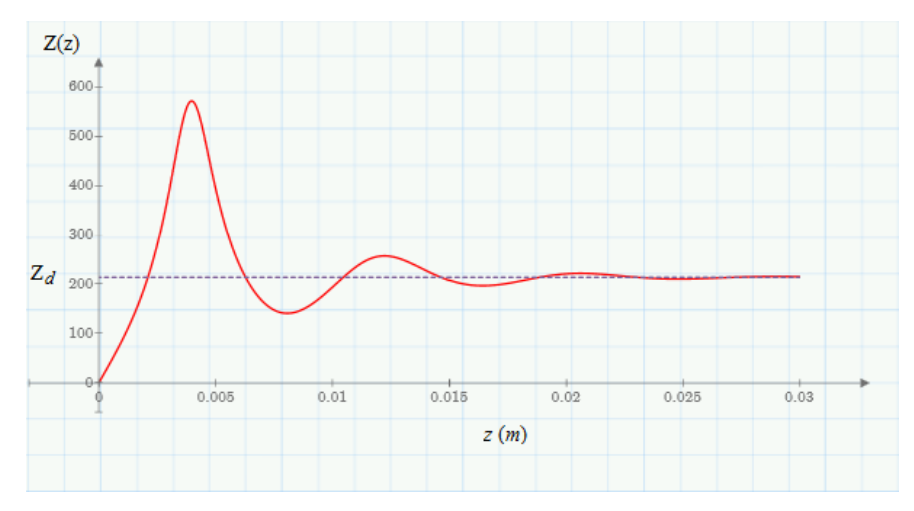

Fig. 6. The lossy medium input impedance versus the medium thickness.

It is obvious in Fig. 6 that if the thickness of the medium reaches some particular value, in our case approximately 0.03 $\mathrm{m}$ the impedance becomes constant and equal to the intrinsic impedance of the lossy medium itself. Physically it means that the wave reflected from the conducting surface is so small that it can be neglected and the reflected from the whole structure is given only by the difference between the intrinsic impedance of the lossy medium and intrinsic impedance of free space. Making the impedance equal to impedance of free space we could remove the reflection at all.

\section{B. The Suggested Coating Material}

Analysing the expression in Equation (9) we can see that using the ordinary lossy medium the above condition can never be satisfied. But it is known that the loss due to the conductivity of the medium is not only possible source of loss and hence of the attenuation in medium. The ferrite-titanate mentioned in [5] could have according to this reference the relative permittivity and relative permeability $\mu_{r}=\varepsilon_{r}=60(2-j)$ which means that intrinsic impedance of such a medium is equal to intrinsic impedance of free space still providing the necessary loss.

As a practical example using the ferrite-titanate material and with the assistance of PTC ${ }^{8}$ MathCAD, the real and imaginary-part of the input impedance at $1 \mathrm{GHz}$ as a function of medium thickness is computed and the relation is shown in Fig. 7.

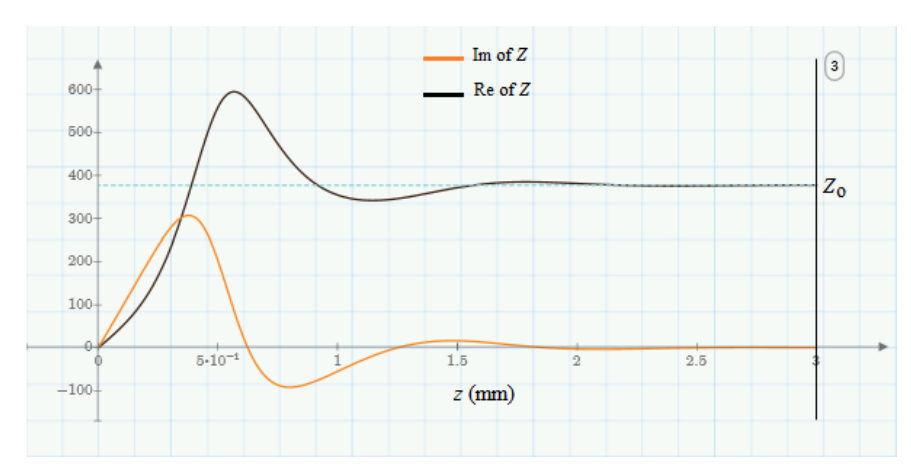

Fig. 7. The relation between the real and imaginary-part of the input impedance and medium thickness.

The above graph shows that the $3 \mathrm{~mm}$ thick layer of the ferrite-titanate material provides at frequency $1 \mathrm{GHz}$ almost exactly the intrinsic impedance of free space so that no reflection should appear.

Let us define the difference between the magnitude of the impedance $Z_{\text {in }}$ of the structure at given thickness say $3 \mathrm{~mm}$ and the free-space impedance for different frequencies.

The diagram in Fig. 8 shows that below the wavelength approximately equal to $30 \mathrm{~cm}$ (above the frequency of $1 \mathrm{GHz}$ ) the reflection for the normal incident wave should be equal to zero.

This frequency-independent technique together with the coating using ferrite-titanate represent a practical solution to reduce the backscattering and be a stealth target against the usual radar bands, which range from $2 \mathrm{GHz}$ up to $40 \mathrm{GHz}$ [4]. 


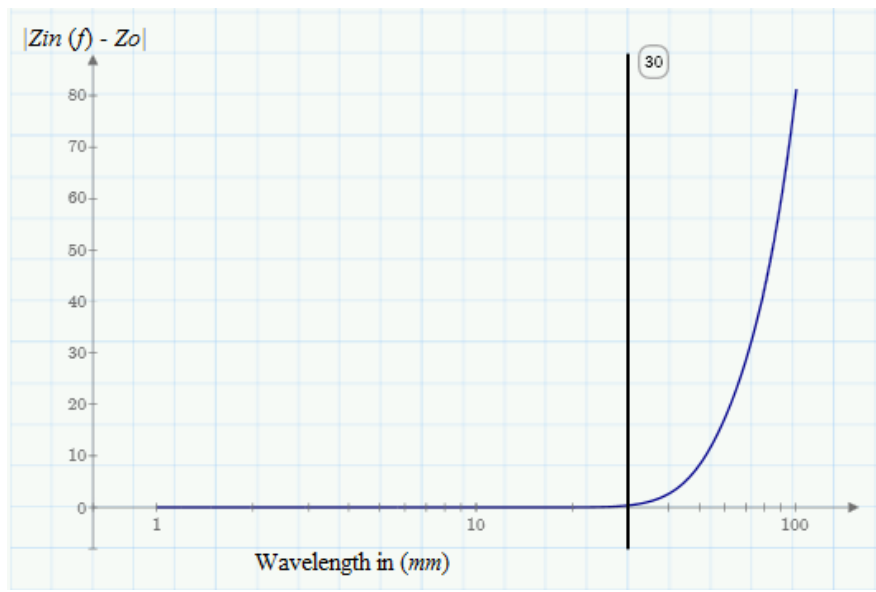

Fig. 8. The relation between the input impedance minus the intrinsic impedance and the wavelength.

For low-frequency radar that uses frequencies lower than 1 $\mathrm{GHz}$, the presented technique is workable but the coating material thickness increases linearly as the frequency decreases, e.g. at a frequency of $100 \mathrm{MHz}$ and above, a 30 $\mathrm{mm}$ coating material of ferrite-titanate is necessary to reduce the backscattering where the reflection should be equal to zero.

\section{CONCLUSION}

A frequency-dependent and frequency-independent stealth coating techniques was presented in this paper. It was found that applying the multi-layer system with using a ferritetitanate coating material of $3 \mathrm{~mm}$ thickness it is possible to reduce the backscattering at frequency of $1 \mathrm{GHz}$ and above, this is normally the frequency band of surveillance and targeting radars.

\section{REFERENCES}

[1] S. Stroobandt, "The Characterization of Surface Waves on LowObservable Structures," M.Sc. Thesis, University of Hull, August 1997.

[2] G.A., Rao and S.P. Mahulikar, "Integrated review of stealth technology and its role in airpower". Aeronautical Journal, vol. 106, no. 1066, pp. 629-641.

[3] D. Howe, "Introduction to the basic technology of stealth aircraft: Part 2 - Illumination by the enemy (active considerations)," Journal of Engineering for Gas Turbines and Power, vol. 113, January 1991, pp. 80-86, Jan 1991.

[4] M. I. Skolnik, Introduction to Radar Systems, Tata McGraw Hill, 2003.

[5] J. D. Kraus, Elecromagnetics, McGraw- Hill, New York, 1992. 